\title{
Tetracycline-Based Binary Ti Vectors pLSU with Efficient Cloning by the Gateway Technology for Agrobacterium tumefaciens-Mediated Transformation of Higher Plants
}

\author{
Seokhyun Lee ${ }^{1}$, Guiying Su${ }^{1}$, Eric Lasserre ${ }^{1,2}$, Norimoto Murai ${ }^{1 *}$ \\ ${ }^{1}$ Department of Plant Pathology and Crop Physiology, Louisiana State University and LSU AgCenter, Baton Rouge, USA; \\ ${ }^{2}$ Laboratoire Genome et Developpement des Plantes, Universite de Perpignan, Perpignan, France. \\ Email: *nmurai@1su.edu
}

Received April 22 $2^{\text {nd }}, 2013$; revised May 22 $2^{\text {nd }}, 2013$; accepted June $15^{\text {th }}, 2013$

Copyright (C) 2013 Seokhyun Lee et al. This is an open access article distributed under the Creative Commons Attribution License, which permits unrestricted use, distribution, and reproduction in any medium, provided the original work is properly cited.

\begin{abstract}
We constructed small high-yielding binary Ti vectors with a bacterial tetracycline resistance gene to facilitate efficient cloning afforded by the Gateway Technology (Invitrogen) for Agrobacterium tumefaciens-mediated transformation of higher plants. The Gateway Technology vectors are kanamycin-based, thus tetracycline-based destination and expression vectors are easily selected for the antibiotic resistance in the Escherichia coli media. We reduced the size of the tetracycline resistance gene TetC from pBR322 to 1468 bp containing 1191 bp of the coding region, 93 bp of 5'-upstream, and 184 bp 3'-downstream region. The final size of binary Ti vector skeleton pLSU11 is 5034 bp. pLSU12 and 13 have the kanamycin resistance NPTII gene as a plant-selectable marker. pLSU14 and 15 contain the hygromycin resistance $H P H$ gene as a selection marker. pLSU13 and 15 also have the $\beta$-glucuronidase (GUS) reporter gene in addition to the plant selection marker. We also constructed a mobilizable version of tetracycline-based binary Ti vector pLSU16 in which the mob function of ColE1 replicon was maintained for mobilization of the binary vector from E. coli to A. tumefaciens by tri-parental mating. The final size of binary Ti vector skeleton pLSU16 is $5580 \mathrm{bp}$. New tetracycline-based binary Ti vectors pLSU12 were found as effective as kanamycin-based vector pLSU2 in promoting a 10 -fold increase in fresh weight yield of kanamycin-resistant calli after A. tumefaciens-mediated transformation of tobacco leaf discs. Using the Gateway Technology we introduced the plant-expressible GUSgene to the T-DNA of binary Ti vector pLSU12. Expression of the $\beta$-glucuronidase enzyme activity was demonstrated by histochemical staining of the GUS activity in transformed tobacco leaf discs.
\end{abstract}

Keywords: Agrobacterium tumefaciens; Binary Ti Vectors; Gateway Technology; pLSU; Tobacco Leaf Disk Transformation; Tetracycline Resistance

\section{Introduction}

Bacteriophage $\lambda$ relies on the site-specific recombination reaction to integrate the phage DNA by the BP clonase into the bacterial chromosome and excise it out by the LR clonase [1]. The BP clonase reaction for DNA integration is catalyzed by the phage integrase and integration host factor. Two attB sites (21 to $25 \mathrm{bp}$ ) at the ends of a target DNA fragment (or a PCR product) recombine with two attP sites of the Gateway donor vector (pDONR), resulting in generation of two at $\mathrm{L}$ sites (96 bp) in an entry vector (pENTR) concomitant with transfer and integration of the target DNA [2]. The LR clonase reaction for DNA excision is catalyzed by the

\footnotetext{
"Corresponding author.
}

phage excisionase, integrase, and integration host factor. Two at $t \mathrm{~L}$ sites flanking the target DNA in the entry vector recombine with two att $\mathrm{R}$ sites of a destination vector (pDEST), resulting in creation of two attB sites in an expression vector (pEXPR) and excision/transfer of the target DNA fragment. Succession of four Gateway vectors, donor, entry, destination, and expression vectors are bacterial kanamycin-based plasmids.

The $\lambda$ clonase recognizes the nine core base sequence 5'-CAACTTNNT-3' at the recombination points of the att $\mathrm{B}$ and $a t t \mathrm{P}$ sites, and also interacts with the eleven base sequences 5'-C/AAGTCACTAT-3' in the P and P' arm of attP site. The recognition sequences for the sitespecific recombination reactions were engineered to create four different variants each of $a t t \mathrm{~B}, \operatorname{att} \mathrm{P}, a t t \mathrm{~L}$ and 
att $\mathrm{R}$ [3]. Thus, att $\mathrm{B} 1$ recombines specifically with att $\mathrm{P} 1$, att $\mathrm{B} 2$ interacts only with $\operatorname{att} \mathrm{P} 2$, att $\mathrm{B} 3$ with att $\mathrm{P} 3$, and att $\mathrm{B} 4$ with att $\mathrm{P} 4$. Four variants of recombination sites became a basis for MultiSite Gateway technology for the directional cloning, reading frame-specific recombination and modular assembly of multiple DNA fragments in a single LR clonase reaction $[4,5]$. This technology enables modular assembly of a promoter, coding, and terminator sequences in the destination vector, selecting from a collection of the multiple sequences in the entry vectors $[6,7]$.

The MultiSite Gateway methodology was introduced to facilitate the predefined assembly of gene sequences in T-DNA of a binary Ti vector for A. tumefaciens-mediated plant transformation [7]. Binary Ti vectors pPZP and pCambia with ColE1 and VS1 replicons were selected for a backbone of the vectors with a pair of any combination of the recombination att $\mathrm{R} 1$, att $\mathrm{R} 2$, att $\mathrm{R} 3$, $a t t \mathrm{R} 4$ and $a t t \mathrm{R} 5$ in the destination vector [5]. Becasue the Gateway entry vectors carry the kanamycin-resistance gene for bacterial selection, the pPZP-based destination vectors with the streptomycin/spectinomycin-resistance gene [4-7] were preferred over the pCambia-based vectors with the kanamycin-resistance gene $[8,9]$. However, the pPZP-based destination vectors are not suited for transformation of A. tumefaciens since the most commonly used strains LBA4404, EHA101 and 105 of $A$. tumefaciens contain the avirulent $\mathrm{Ti}$ plasmid with the streptomycin-resistance marker. In addition, some strains of A. tumefaciens are reported to be resistant to low levels of spectinomycin [10]. Thus, the introduction of pPZP vectors to A. tumefaciens is not assured using simple antibiotic selection for streptomycin or spectinomycin, and needs additional steps to circumvent the difficulty. This provides a practical advantage for using the tetracycline-based pLSU binary vectors as destination vectors for efficient cloning of multiple fragments to create expression vectors. The C58 strain of A. tumefaciens has a tetracycline-resistance determinant and is resistant to low levels of tetracycline [10].

The objective of this research is to develop new tetracycline-based binary $\mathrm{Ti}$ vectors to facilitate efficient cloning by the Gateway Technology. The binary vectors will be tested for transformation of tobacco leaf discs and for expression of the $\beta$-glucuronidase GUS reporter gene.

\section{Materails and Methods}

\subsection{Chemicals and Enzymes}

Antibiotics (ampicillin, carbenicillin, gentamycin, kanamycin, rifampicin, streptomycin, spectinomycin, tetracycline) and other chemicals used in this experimentwere purchased mainly from Sigma-Aldrich (St. Louis, MO).
Restriction endonucleases (AvrII, BamHI, BsrGI, BstBI, EcoRI, EcoRV, HindIII, KpnI, MfeI, NheI, PstI, PvuII, SalI, ScaI, SphI, XbaI and XhoI), Deep Vent DNA polymerase, and T4 DNA ligase were purchased from New England Biolab (NEB; Beverly, MA) and/or Bethesda Research Laboratory (BRL; Grand Island, NY). The lysozyme was purchased from Sigma and Ultra Pfu DNA polymerasewas from Stratagene (La Jolla, CA). The enzymes were treated as instructed by suppliers.

\subsection{Bacterial Strains and Plasmid DNA}

The XL1Blue-MR strain was purchased from Stratagene (La Jolla, CA). The MR strain has no antibiotic resistance since the F' episome was eliminated while the XL1Blue and XL2Blue strains are tetracycline-resistant. The genotype of the MR strain is as follows: $\operatorname{rec} A 1$, endA1, supE44, relA1, $\Delta(m c r A) 183, \Delta(m c r C B-h s d S M R$ $m r r) 173$, gyrA96, thi-1. The EndA $A^{-}$phenotype of XL1Blue-MR strain allows to yield high quality plasmid DNA. The A. tumefaciens strain LBA4404 has TiAch5 chromosome which contains rifampicin resistance gene and disarmed Ti plasmid pAL4404 with spectinomycinand streptomycin-resistance genes [11]. The final concentrations of spectinomycin and streptomycin for selection are $100 \mathrm{mg} / \mathrm{L}\left(\mathrm{Sp}^{100}\right)$ and $50 \mathrm{mg} / \mathrm{L}\left(\mathrm{St}^{50}\right)$, respectively. A. tumefaciens was grown on Agrobacterium media (A. media) containing $2.0 \mathrm{~g}$ mannitol, $2.0 \mathrm{~g}\left(\mathrm{NH}_{4}\right)_{2} \mathrm{SO}_{4}$, $5.0 \mathrm{~g}$ yeast extract, and $100 \mathrm{ml}$ of $10 \times$ salt solution per liter. For the $10 \times$ salt solution, $109.0 \mathrm{~g} \mathrm{KH}_{2} \mathrm{PO}_{4}, 1.6 \mathrm{~g}$ $\mathrm{MgSO}_{4} \cdot 7 \mathrm{H}_{2} \mathrm{O}, 0.05 \mathrm{~g} \mathrm{FeSO}_{4} \cdot 7 \mathrm{H}_{2} \mathrm{O}, 0.11 \mathrm{~g} \mathrm{CaCl}_{2} \cdot 2 \mathrm{H}_{2} \mathrm{O}$, and $0.02 \mathrm{~g} \mathrm{MnCl}_{2} \cdot 4 \mathrm{H}_{2} \mathrm{O}$ were dissolved in one liter of $\mathrm{H}_{2} \mathrm{O}$, and the $\mathrm{pH}$ of the solution was adjusted to 7.0 with $1.0 \mathrm{~N} \mathrm{KOH}$. After making a volume to one liter, the $10 \times$ salt solution was heated to boil and the precipitates were filtered through Whatman No. 1 filter paper. After the $10 \times$ salt solution was added the $\mathrm{pH}$ of the media was adjusted to 7.0 prior to autoclaving. Transformation of $A$. tumefaciens was conducted in YEB media (Sucrose $5 \mathrm{~g}$, Bacto-Peptone $5 \mathrm{~g}$, Beef Extract $5 \mathrm{~g}$, Yeast Extract $1 \mathrm{~g}$, $0.002 \mathrm{M} \mathrm{MgSO}_{4}$ per liter). Magnesium ion was omitted from the media when tetracycline was used for bacterial selection.

Plasmids used for experiments were pBR322 [12-14], pUC19 [15,16], pCAMBIA1301purchased from Cambia (http://www.cambia.org.au, Canberra, Australia), pBluescriptII KS(+) from Stratagene (La Jolla, CA) and pUC4KIXX and -KSAC from Pharmacia (Uppsala, Sweden) [17].

\subsection{Oligodeoxyribonucleotides and Plasmid DNA Manipulation}

Oligonucleotides used for PCR, mutagenesis or DNA 
sequencing were custom-ordered and synthesized by Sigma-Aldrich (St. Louis, MO). Plasmid DNA was isolated by alkalinelysis method [18] and purified by $\mathrm{CsCl}_{2}-$ EtBr gradient centrifugation method [19]. The GENECLEAN kit purchased from BIO101 (Carlsbad, CA) was used to extract DNA from agarose gel [20]. QuickChange Multi Site-Directed Mutagenesis Kit was obtained from Stratagene. Other molecular cloning methods were according to Sambrook and Russell [21].

\subsection{Construction of Tetracycline-Based Binary Ti Vectors pLSU12}

New binary Ti vectors pLSU11 to 16 have the tetracycline resistance gene $\operatorname{Tet} C$ as a bacterial selection marker. The TetC gene was amplified from pBR322 and modified to eliminate fiverestriction enzyme sites, two NheI sites, one each of EcoRV, SphI, and SalI sites. The primers used for the mutagenesis were described in details in the Ph.D. thesis of S. Lee [22]. The mutagenesis reactions yielded 5429 bp of pBRVS2 $\triangle$ NENSS.

The modified $\operatorname{Tet} C$ gene was amplified by PCR from pBRVS2 $\triangle$ NENSS using two primersTet-F2 and Tet-R1 [22]. Both primers have misplaced bases to introduce NheI sites at both ends of the amplified TetC gene (1468 bp). The amplified $\operatorname{Tet} C$ gene replaced the NPTI gene in binary vectors pLSU2 and 4 . To remove the NPTI gene from the binary vectors, two primers were designed to perform a reverse PCR. Both primers have misplaced bases to introduce AvrII sites atoutside of NPTI gene to be removed, and amplified PCR products, pLSU2 $\triangle$ NPTIand pLSU4 $\triangle$ NPTI. The amplified 1468 bp of TetC gene was ligated into the binary vectors without the NPTI gene resulting in new binary vectors pLUS12 and pLSU14 with tetracycline resistance for bacterial selection (6412 and $6648 \mathrm{bp}$, respectively) (Figures 1 and 2).

The $\beta$-Glucuronidase GUS gene was amplified from pCAMBIA1305.2 with primer 1305-1F2 and 1305-1R2 [22]. Both primers introduced new HindIII sites at the ends of GUS fragment including CaMV35S promoter, GUS gene with His6, glycine-rich protein signal peptide, catalase intron and nopaline terminator. After HindIII digestion, the amplified 3007 bp fragment were introduced into the expression vectors pLSU12 and pLSU14 at the HindIII site 3'-adjacent to the Hph or NPTII gene producing 9419 bp of pLSU13 and 9655 bp of pLSU15, respectively (Figure 2).

\subsection{DNA Sequencing of pLSU12}

DNA sequences of two strands of new binary vectors pLSU12 were determined with an Applied Biosystems ${ }^{\mathrm{TM}}$ 3730xl DNA Analyzerat Eurofins MWG Operon (Huntsville, AL). Twenty eight sequencing primers were de- signed [22], and synthesized by Sigma. The complete DNA sequence of tetracycline-based binary vector skeleton (pLSU11) is submitted to GenBank (Submission \#1398415). In the tetracycline resistance gene at $1142 \mathrm{bp}$, the nucleotide $\mathrm{C}$ was confirmed as $\mathrm{T}$, and in the termination region of $T e t^{R}$ gene at 1425 bp GCGG were missing from the pBR322 sequence listed in GenBank. $C$ was inserted at $1467 \mathrm{bp}$, the ligation junction between $T e t^{R}$ gene and ColE1 replicon. The unexpected $\mathrm{G}$ in the RepA region at $3376 \mathrm{bp}$ and the insertion of $16 \mathrm{bp}$-long fragment CGCGCGGACAAGCTAG in the termination region of $T e t^{R}$ gene at the ligation junction between VS1 replicon and T-DNA region were determined so as in the sequence of pLSU4.

\subsection{Mobilizable Tetracycline-Based Binary Vector pLSU16}

The ColE1 replicon and tetracycline resistance gene of pBR322 [13] were amplified as a template for the mobilizable binary Ti vector pLSU16. The ampicillin resistanceand ROP genes were excluded by two separate amplification reactions of the pBR322 template, and unique HindIII and BamHI sites of pBR322 were eliminated

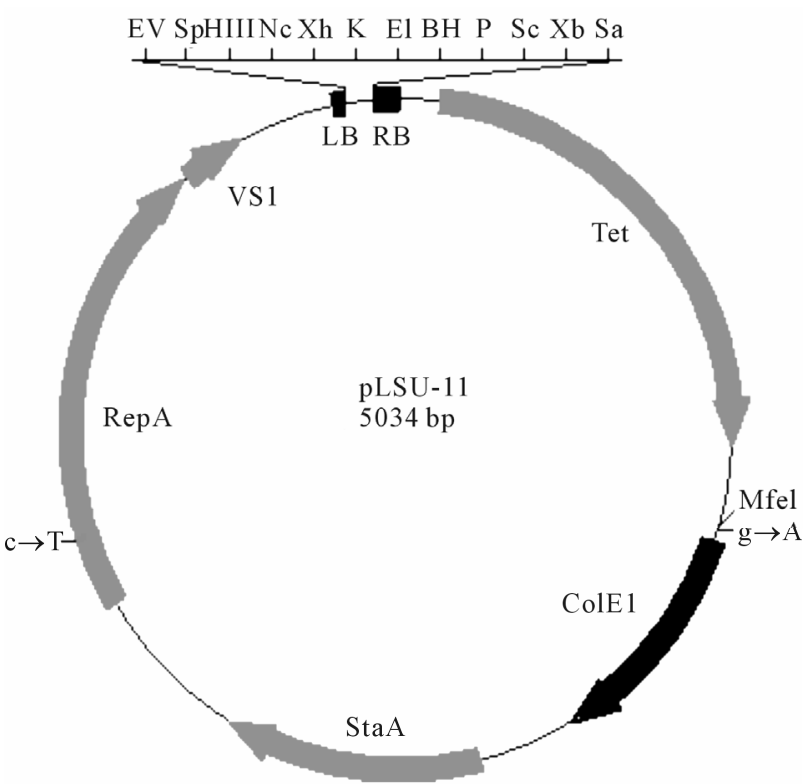

Figure 1. Schematic presentation of backbone structure of tetracycline-based binary Ti vector pLSU11 (5034 bp). T-DNA is at the top of figure limited by the right (RB) and left border (LB) with 12 common restriction endonuclease sites, EcoRV (EV), SphI (Sp), HindIII (HIII), NcoI (Nc), XhoI (Xh), KpnI (K), EcoRI (EI), BamHI (BH), PstI (P), ScaI (Sc), XbaI (Xb), and SacI (Sa). The backbone plasmid includes the tetracycline resistance gene $\left(T e t^{R}\right)$, ColE1 origin of replication from pUC19 (ColE1), Stability region A (StaA), Replication region A (RepA), and VS1 origin of replication (VS1). 


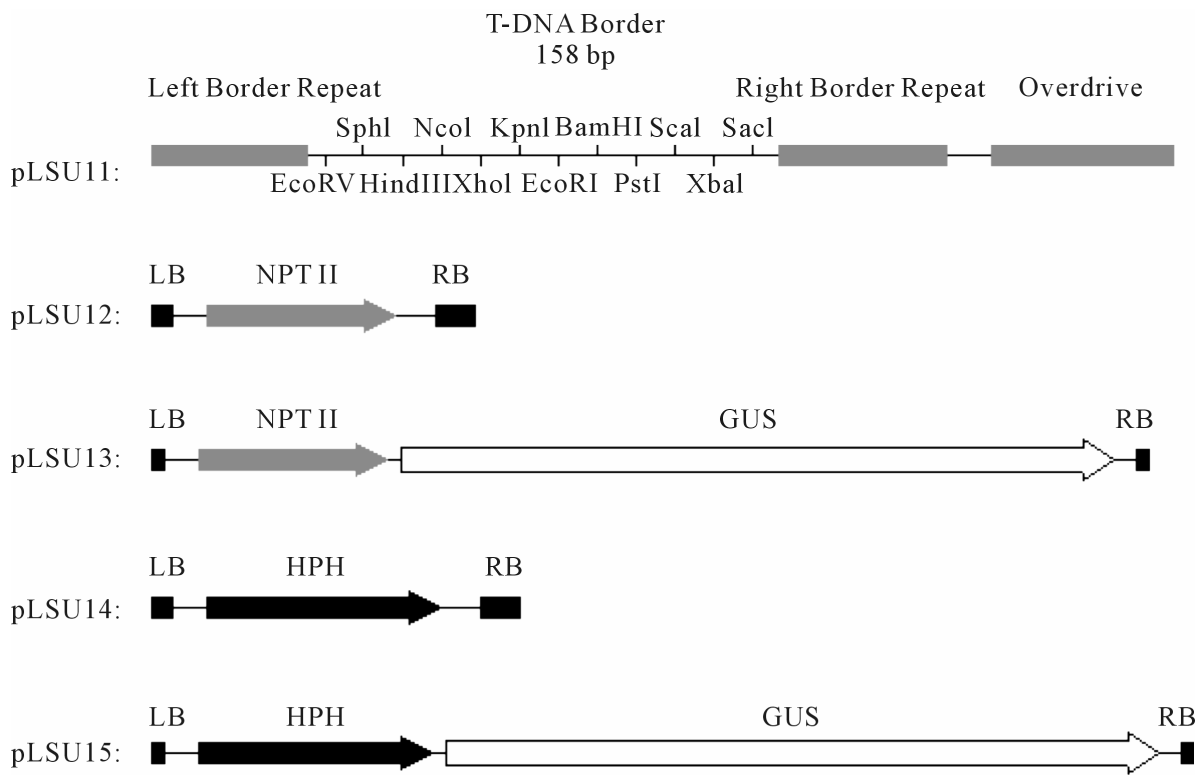

Figure 2. Schematic presentation of the T-DNA region of tetracycline-based binary Ti vectors pLSU11 to 15 . pLSU-11 is a basic skeleton vector with the twelve common restriction sites in T-DNA. pLSU12 and 13 have the Neomycin PhosphoTransferase II gene (NPTII) adjacent to the left border as a plant selection marker for kanamycin resistance. pLSU14 and 15 contain the Hygromycin B Phosphotransferasae gene $(\mathrm{HPH})$ adjacent to the left border as a plant selection marker for hygromycin resistance. pLSU13 and 15 also include the $\beta$-glucuronidase reporter gene $(G U S)$ in addition to the plant selection marker in the T-DNA.

yielding 2243 bp pORItet [22]. First, theunique HindIII site at 29 to $34 \mathrm{bp}$ of pBR322 was eliminated by HindIII digestion and filled-in by Klenow fragment producing pBR322-dHindIII. The region from the tetracycline resistance gene to ColE1 replicon was amplified with primers BR322-1 and BR322-2producinga smaller 3206 bp pBR-d1. Next, the unique BamHI site in tetracycline resistance gene was inactivated by making a single point mutation using primers BR322-5 and BR322-6. Finally, new PvuII and BstBI sites were introduced by making two single point mutations on 1408 to $1413 \mathrm{bp}$ and 2396 to $2401 \mathrm{bp}$, and $R O P$ gene was removed with primers BR322-3 and BR322-4yielding pORItet.

The broad host range replication origin and stability region was amplified from a binary vector pGV941 using primers with new restriction endonuclease sites EcoRI and MfeI. The amplified fragment digested with both restriction enzymes was ligated into $2241 \mathrm{bp}$ to $3 \mathrm{bp}$ EcoRI site of pORItet producing 5429 bppBRVS1 [22]. The new $M f e I$ sites of the PCR fragment were inactivated by ligation to the EcoRI site of pORItet due to the compatible cohesive end of MfeI to EcoRI.

The T-DNA left and right border sequences originated from the octopine-type Ti plasmid pTi15955 were cloned from pKSLR [22]. Due to the short length of the T-DNA border sequences in pKSLR, it was necessary to make sure that only a single copy of the left/right border sequences are properly inserted into pBRVS1. For this purpose, the kanamycine resistance phenotype was introduced to the binary vector, from the neomycin phosphotransferase II (NPTII) gene of transposon Tn5. Plasmid pUC4-KIXX (Pharmacia) was digested with HindIII and the $1568 \mathrm{bp}$ fragment containing NPTIIgene was inserted into HindIII site of pKSLR. This plasmid was named as pLRKIXX and the colonies were double-selected for kanamycin and ampicillin resistance. After kanamycin resistance selection, the MfeI-LB-Kan ${ }^{\mathrm{R}}$ MCS-RB-MfeI fragment was cut with $\mathrm{MfeI}$ and inserted into EcoRI site of pBRVS1. This kanamycin resistance gene was removed by HindIII digestion after the insertion of single copy of T-DNA border into pBRVSI was confirmed. The final product was named aspLSU16 (Figure 3).

\subsection{Gateway Technology}

The three reading frame cassettes (1711 bp of RfA, 1713 bpof RfB, and 1714 bp of RfC1; Invitrogen (Carlsbad, $\mathrm{CA})$ have the suicidal $c c d \mathrm{~B}$ gene for inhibiting the DNA gyrase activity and chloramphenicol resistance gene $\left(C m^{R}\right)$ flanked by $a t t \mathrm{R} 1$ and attR2 sites. AScaI site (Sc) adjacent to the T-DNA right border of the binary vector pLSU12 was used for cloning site of Gateway ${ }^{\circledR}$ reading frame cassette (Figures 1 and 2). First, the Scalsite in pLSU12 was digested and dephosphorylated by calf intestinal alkaline phosphatase (CIAP). Then the dephosphorylated vector was ligated with Gateway ${ }^{\circledR}$ cassettes 


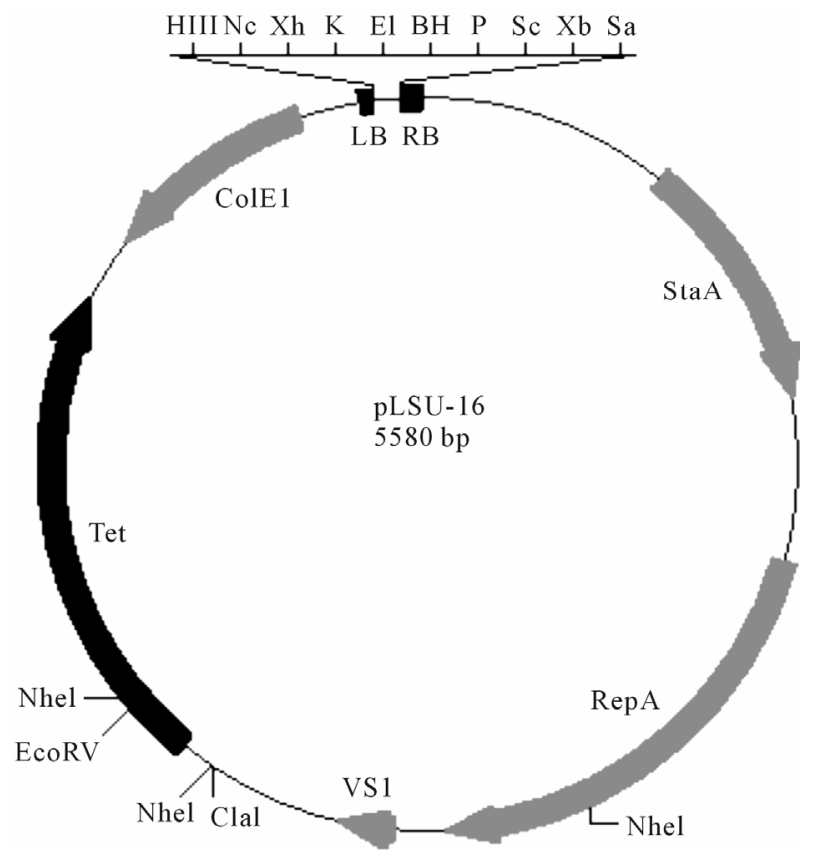

Figure 3. Schematicpresentation of mobilizable binary $\mathrm{Ti}$ vector pLSU16 (5580 bp). T-DNA is at the top of figure limited by the right (RB) and left border (LB) with 12 common restriction endonuclease sites, $\operatorname{EcoR} V(\mathrm{EV}), \operatorname{SphI}$ (Sp), HindIII (HIII), NcoI (Nc), XhoI (Xh), KpnI (K), EcoRI (EI), BamHI (BH), PstI (P), ScaI (Sc), XbaI (Xb), and SacI (Sa). The backbone plasmid includes the tetracycline resistance gene $\left(\mathrm{Tet}^{R}\right)$, ColE1 origin of replication from pBR322 (ColE1), Stability region A (StaA), Replication region A (RepA), and VS1 origin of replication (VS1).

RfA, RfB, and RfC1, respectively, producing pLSU17A, 17B, and 17C1 (Figure 4). The ligation products were transformed into $E$. coli strain of the One shot ${ }^{\circledR} c c d \mathrm{~B}$ Survival $^{\mathrm{TM}} 2 \mathrm{~T} 1^{\mathrm{R}}$ competent cells with gyrA mutation. Colonies were selected on LB agar plates for $30 \mathrm{mg} / \mathrm{L}$ chloramphenicol and $10 \mathrm{mg} / \mathrm{L}$ tetracycline under dark. The insertion of Gateway ${ }^{\circledR}$ cassette was determined by BsrGI restriction enzyme whose recognition site is located in the att $\mathrm{R} 1$ and $a t t \mathrm{R} 2$ sites.

The plant-expressible $\beta$-Glucuronidase (GUS) gene was amplified by PCR from pCAMBIA1305.2, using two primers containing $a t t \mathrm{~B} 1$ and $a t t \mathrm{~B} 2$ sites at the ends. A donor vector pDONR 221 has the $c c d \mathrm{~B}$ and $\mathrm{Cm}^{R}$ gene flanked by the attP1 and attP2 sites. BP Clonase II enzyme catalyzed the $\mathrm{BP}$ recombination reaction between the attB sites of the GUS gene and the attP sites of pDONR 221. After incubation for one hour at $25^{\circ} \mathrm{C}$, the Proteinase $\mathrm{K}$ solution was added and incubated for 10 min at $37^{\circ} \mathrm{C}$. The reaction products were transformed in E. coli TOP10/P3 One Shots and selected for kanamycinat $50 \mathrm{mg} / \mathrm{L}$. Colonies were picked and transferred to a replica plate under selection of chloramphenicol $30 \mathrm{mg} / \mathrm{L}$ and kanamycin $50 \mathrm{mg} / \mathrm{L}$. If the site-specific recombina- tion happened between attB1/B2 and att $\mathrm{P} 1 / \mathrm{P} 2$ sites, the replica colonies are sensitive to chloramphenicol selection because the $C m^{R}$ gene was removed by recombination, yielding a new entry vector pENTR-GUS.

The destination binary vectorpLSU17A was mixed with pENTR-GUS and Gateway ${ }^{\circledR}$ LR Clonase ${ }^{\mathrm{TM}}$ II enzyme mix, and the LR recombination reaction was performed at $25^{\circ} \mathrm{C}$ overnight. After the Proteinase $\mathrm{K}$ treatment the reaction products were transformed in $\mathrm{E}$. coli TOP10/P3. Selection for tetracycline resistance at 10 $\mathrm{mg} / \mathrm{L}$ and for chloramphenicol sensitivity at $30 \mathrm{mg} / \mathrm{L}$ yielded colonies containing the expression vector pLSU17A-GUS.

\subsection{Freeze-Thaw Transformation of A. tumefaciens}

Cells of $A$. tumefaciens LBA4404 strain were grown at $28^{\circ} \mathrm{C}$ in YEB media. Cells were prepared as described by Hofgen and Willmitzer [23] and as modified as follow. Ten $\mathrm{ml}$ of overnight culture were mixed with $50 \mathrm{ml}$ of fresh YEB media and incubated at $250 \mathrm{rpm}$ for six to seven hours until $A_{600}$ reached at 0.5. After cooling on ice for $30 \mathrm{~min}$, cells were harvested by centrifugation at $3000 \mathrm{~g}$ for $20 \mathrm{~min}$ at $4^{\circ} \mathrm{C}$. Cell pellet was washed once in $30 \mathrm{ml}$ of TE buffer and re-suspended in $1 \mathrm{ml}$ of YEB media. One $\mu \mathrm{g}$ of DNA was mixed with $100 \mu \mathrm{l}$ of cells, and frozen in an ethanol bath at $-80^{\circ} \mathrm{C}$ for two hours to overnight. The frozen cell DNA mixture was thawed at $37^{\circ} \mathrm{C}$, mixed with $1 \mathrm{ml}$ of YEB media and incubated at $28^{\circ} \mathrm{C}$ with gentle rotation at $150 \mathrm{rpm}$ for five hours for stabilization. Aliquots of $100 \mu \mathrm{l}$ were plated on YEBagar media containing appropriate antibiotics and incubated at $28^{\circ} \mathrm{C}$ for two to three days.

\subsection{Tobacco Leaf Disc Transformation Mediated by $A$. tumefaciens}

A. tumefaciens-mediated transformation of tobacco leaf disc was performed as described by Su et al. [24].

\subsection{Histochemical Detection of $\beta$-Glucuronidase Activity}

The $\beta$-glucuronidase activity was detected after histochemical staining of $A$. tumefaciens-infected leaf disc by 5-bromo-4-chloro-3-indolyl $\beta$-D-glucuronide (X-GlcA).

\section{Results}

\subsection{Tetracycline-Based Binary Ti Vectors pLSU12 and 14}

We constructed new tetracycline-basedbinary Ti vectors by replacing the bacterial kanamycinresistance gene of binary vectors pLSU2 and 4 [25] with the tetracycline 


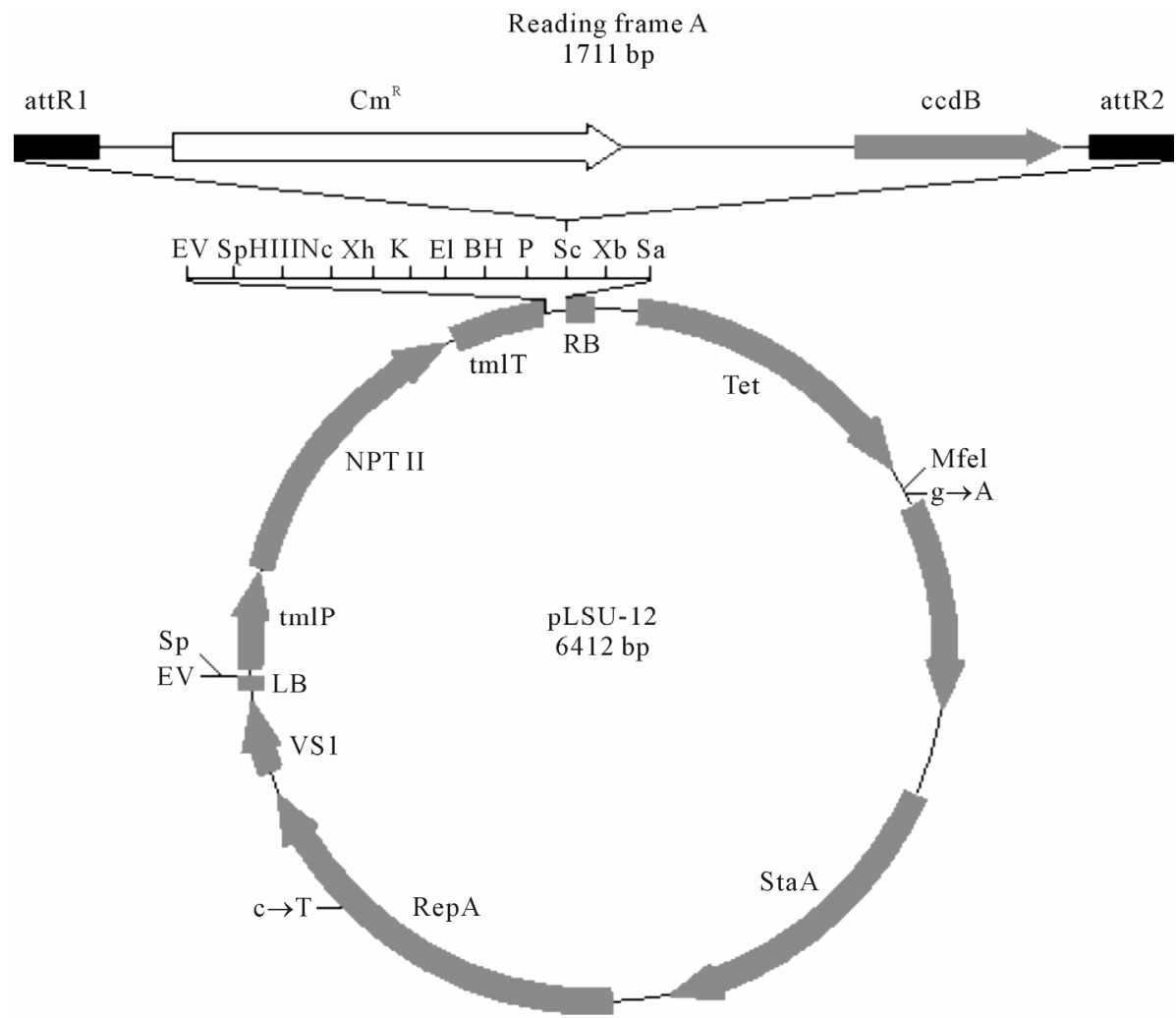

Figure 4. Schematic presentation of Gateway destination vector pLSU17A. At the top is the reading frame A fragment with two clonase recognition sites (attR1 and attR2), chloramphenicol resistance gene $\left(\mathrm{Cm}^{R}\right)$, and $c c d B$ gene that inhibits the DNA gyrase (topoisomerase II). T-DNA is limited by the right (RB) and left border (LB) with 12 common restriction endonuclease sites, EcoRV (EV), SphI (Sp), HindIII (HIII), NcoI (Nc), XhoI (Xh), KpnI (K), EcoRI (EI), BamHI (BH), PstI (P), ScaI (Sc), $\mathrm{XbaI}(\mathrm{Xb})$, and $\mathrm{SacI}(\mathrm{Sa})$. The kanamycin resistance gene $\mathrm{NPTII}$ of pLSU12 is located between the SphI and HindIII sites. The backbone plasmid includes the tetracycline resistance gene $\left(T e t^{R}\right)$, ColE1 origin of replication (ColE1), Stability region A (StaA), Replication region A (RepA), and VS1 origin of replication (VS1).

resistance gene, forming pLSU12 and 14, respectively) (Figures 1 and 2). The minimal requirement for the component of tetracycline resistance gene was tested by the tetracycline-resistance comparison and the plasmid stability experiment in E. coli and A. tumefaciens.

\subsection{Bacterial Tetracycline Resistance Gene}

We used the tetracycline resistance gene TetC from pBR322 for tetracycline selection of bacteria [26]. Tetracycline is a very effective antibiotics since the optimal concentrations for E. coli and A. tumefaicnes are 10 and $2 \mathrm{mg} / \mathrm{L}$, respectively. However, there are some limitations in use of the antibiotics because tetracycline is light-sensitive, and is inhibited by magnesium ion included incommonly used bacterial media. The XL1BlueMR strain of $E$. coli was used since it has no antibiotic resistance without the F' episome while the XL1Blue and XL2Blue strains are tetracycline-resistant.

Fiverestriction enzyme sites for two NheI sites, one each of EcoRV, SphI, and SalI site were eliminated from the TetC coding and 5'-upstream regions by single point mutations without alternation of the amino acid codons, so that these restriction sites remain unique in the multicloning site of T-DNA. Based on the sequence analysis of $T e t C$ gene, we deduced the minimal size of gene extending from the 5'-upstream region including -35 and -10 elements to the 3 '-downstream region following the small stem-loop structures presumably acting as a transcription termination signal. The new truncated TetC gene of $1468 \mathrm{bp}$ contains $1191 \mathrm{bp}$ of the coding region with $93 \mathrm{bp}$ of 5'-upstream region to the initiation codon, and $184 \mathrm{bp} \mathrm{3'-downstream} \mathrm{from} \mathrm{the} \mathrm{termination} \mathrm{codon.}$ This truncated gene confers the resistance up to 100 $\mathrm{mg} / \mathrm{L}$ of tetracycline as effective as the wild-type gene, but less effective at $200 \mathrm{mg} / \mathrm{L}$ in E. coli. The truncated $T e t C$ gene was used to replace the bacterial kanamycin resistance NPTI gene from binary vectors pLSU2 and 4, generating pLSU12 and 14 [22].

DNA sequence analysis of pLSU12 indicated that all single point mutations introduced to the tetracycline resistance $\left(T e t^{R}\right)$ gene were confirmed as expected. How- 
ever, we found one and $16 \mathrm{bp}$ insertions at the junctions of ligation reactions and $4 \mathrm{bp}$ deletion in the tetracycline resistance gene as noted in Materials and Methods. DNA sequence of the binary vector skeleton pLSU11 was deposited to GenBank at submission number 1398415.

\subsection{Mobilizable Tetracycline-Based Binary Ti Vector pLSU16}

We also constructed amobilizable version of tetracylinebased binary Ti vectors pLSU16 in which the mob function of ColE1 replicon was maintained for mobilizationfrom $E$. coli to A. tumefaciens by tri-parental mating assisted by pRK2013. The final size of vector skeleton pLSU16 is 5580 bp long consisting of the $T c$ gene, ColE1 and VS1 replicons, and T-DNA (Figure 3).

\subsection{A. tumefaciens-Mediated Transformation of Tobacco Leaf Discs}

The tetracycline-based binary Ti vector pLSU12 in $A$. tumefaciencs was used for transformation of tobacco leaf discs after four-day co-cultivation. Transformed leaf discs were selected for in the presence of $300 \mathrm{mg} / \mathrm{L}$ of kanamycin for four weeks. Stable expression of introduced kanamycin-resistance gene was evident by up to 10 -fold increase in fresh weight yield in $\mathrm{g}$ of treated tobacco leaf discs (Table 1). The tetracycline-based pLSU12 was as effective as the kanamycin-based pLSU2 in the growth promotion assay.

\subsection{Gateway Technology Expression Vector with GUS}

The tetracycline-based binary vector pLSU12 was used to generate Gateway expression vectors. Three different reading frame cassettes flanked by attR1 and att 2 sites (1711 bp of RfA, 1713 bp of RfB, and 1714 bp of RfC1) were ligated to the ScaI site (Sc) of T-DNA of pLSU12, producing Gateway destination vectors, pLSU17A, 17B, and $17 \mathrm{C} 1$ (Figure 4). Each reading frame cassettes contain the chloramphenicol resistance gene $\left(\mathrm{Cm}^{R}\right)$ and the suicidal $c c d \mathrm{~B}$ gene inhibiting the DNA gyrase activity (topoisomerase II). The ligation products were transformed to the E. coli strain DB3.1 containing gyrA mutation, and the Gateway destination vectors were isolated from colonies after simple selection for tetracycline and chloramphenicol resistance. Aplant-expressible $\beta$-glucuronidase (GUS) gene in a entry vector was used to replace the $\mathrm{Cm}^{R}$ and $c c d \mathrm{~B}$ gene in the destination vector using the LR clonase-catalyzed recombination reaction of the att $\mathrm{L} 1 / a t t \mathrm{~L} 2$ sites of the donor vector with the att $\mathrm{R} 1 /$ att $\mathrm{R} 2$ sites of the destination vector. A pLSU expression vector with the GUS gene was isolated from $E$. coli colonies by simple selection for tetracycline resis-
Table 1. Tetracycline-based binary Ti vector pLSU12 was compared with kanamycin-based binary vectors pLSU2 in its effect on the increase in final fresh weight yield of kanamycin-resistant calli of tobacco leaf disks. Leaf disks were co-cultivated for four days with $A$. tumefaciens strain LBA4404 with or without new binary Ti vector pLSU. Leaf disks were selected at $25^{\circ} \mathrm{C}$ on shoot medium containing 300 $\mathrm{mg} / \mathrm{L}$ of kanamycin and $500 \mathrm{mg} / \mathrm{L}$ of carbenicilin for two weeks. Fresh medium was prepared for additional two weeks of selection. Co-cultivation was performed from 1/11 to $1 / 15$, the first selection from $1 / 15$ to $1 / 29$ and the second selection from $1 / 29$ to $2 / 12 / 2010$. Each treatment had five plates with 10 leaf disks per plate. Numbers in parentheses indicate standard deviations.

\begin{tabular}{cccc}
\hline $\begin{array}{c}\text { pLSU binary } \\
\text { Ti vectors }\end{array}$ & $\begin{array}{c}\text { Plate average } \\
\text { of FW yield } \\
\text { in g }\end{array}$ & $\begin{array}{c}\text { Increase in FW } \\
\text { in g per plate } \\
\text { over vector-less } \\
\text { control }\end{array}$ & $\begin{array}{c}\text { \% leaf discs } \\
\text { with increased } \\
\text { FW }\end{array}$ \\
\hline $\begin{array}{c}\text { Vector-less } \\
\text { control }\end{array}$ & $0.91(0.20)$ & 0 & 0 \\
pLSU2 & $12.06(5.57)$ & $10.96(5.54)$ & 92 \\
pLSU12 & $8.21(1.48)$ & $7.09(1.48)$ & 98 \\
pLSU12-long & $11.24(7.23)$ & $10.12(7.23)$ & 96 \\
\hline
\end{tabular}

tance and chloramphenicol sensitivity.

\subsection{GUS Reporter Gene Expression in Tobacco Leaf Discs}

The plant-expressible GUS gene in the T-DNA of binary vector pLSU17Awas introduced to tobacco after $A$. $t u$ mefaciens-mediated transformation. Expression of the GUS gene was demonstrated by histochemical staining of GUS activity in transformed tobacco leaf discs.

\section{Discussion}

We previously constructed a series of kanamycin-based binary Ti vectors pLSU1 to 5 to improve the transformation frequency and plasmid yield in E. coli and A. tumefaciens for A. tumefaciens-mediated transformation of higher plants $[22,25]$. Transcriptional direction of STA/ REP replicon for A.tumefaciens can be the same as that of ColE1 replicon for E. coli (co-directional transcription), or opposite (head-on transcription) as in the case of widely used vectors (pPZP or pCambia). New binary $\mathrm{pLSU}$ vectors with co-directional transcription yielded in E. coli up to four-fold higher transformation frequency than those with the head-on transcription. Here we converted these kanamycin-based vectors to the tetracyclinebased binary vectors pLSU11 to 15 to exploit the userfriendly features of the Gateway ${ }^{\circledR}$ Technology for efficient cloning. With further introduction of Multi-site Gateway methodology, different combinations of attR 1 , 
attR2, attR3, attR4, attR5 will be inserted in T-DNA region of pLSU and the high-throughput modular assembly of promoter, terminator, and coding region of target gene will be suitable for global analysis of plant gene functions in a genomic scale.

The Gateway Technology relies on use of four kanamycin-based plasmid vectors in quick succession from the donor, entry, destination to expression vectors. Many destination vectors for transformation of higher plants used as a vector skeleton pCambia, pGreen, or pBin19 which has a bacterial kanamycin-resistance gene $[8,9,27]$. The use of the same kanamycin-based vectors made impossible the simple antibiotic selection of coloniesto distinguish the destination vectors from donor/entry vectors after LR clonase reaction. To overcome this difficulty, the entry vector should be linearized before LR recombination or the proper expression vector should be selected based on the plasmid DNA size or restriction enzyme sites bylabor-intensive DNA purification. An alternative approach used the suicidal characteristic of $c c d \mathrm{~B}$ gene in destination binary vectors [28]. After LR recombination reaction the $E$. coli transformants only have either the proper expression vector or unreacted entry vector because transformants harboring unreacted destination vector or entry vector with recombined chloroamphenicol resistance gene and $c c d \mathrm{~B}$ gene cannot survive due to the activation of $c c d \mathrm{~B}$ gene. Thus, the plasmid DNA isolated from the survived clones should be the mixture of entry vector and expression binary vector. After transformation to A. tumefaciens with the mixed plasmid, the transformants harboring the entry vector which does not have replication origin for $A$. tumefaciens cannot survive. The survived colony harboring the proper expression vector can be further used for plant transformation. However, the identity of expression binary vector generated by this method might not easily verified since it is difficult to purify plasmid DNA from A. tumefacins.

The other major group of destination vectors is based on pPZP200 vector which has streptomycin/spectinomycin resistance gene [4-6,29]. The streptomycin selection is often not suitable for Agrobacteria-mediated transformation because the widely used $A$. tumefaciens strain LBA4404 has the streptomycin/spectinomycinresistance gene in the avirulent $\mathrm{Ti}$ plasmid, although these streptomycin selectable vectors can be used for plant transformation using particle bombardment method or Agrobacteria-mediated transformation using other $A$. tumefaciens kanamycin-resistant strain, EHA101.

The new tetracycline-based, Gateway-compatible binary vectors $\mathrm{pLSU}$ are more user-friendly in this aspect. With further introduction of Multi-site Gateway methodology, the high-throughput modular assembly of pro- moter, terminator, and coding region of target gene will be suitable for global analysis of plant gene functions in a genomic scale.

\section{Acknowledgements}

The authors wish to acknowledge the financial support partly from the College of Agriculture, Louisiana State University and LSU AgCenter to N. Murai.

\section{REFERENCES}

[1] A. Landy, "Dynamic, Structural, and Regulatory Aspects of Site-Specific Recombination," Annual Review of Biochemistry, Vol. 58, 1989, pp. 913-949.

doi:10.1146/annurev.bi.58.070189.004405

[2] J. L. Hartley, G. F. Temple and M. A. Brasch, "DNA Cloning Using in Vitro Site-Specific Recombination," Genome Research, Vol. 10, 2000, pp. 1788-1795. doi:10.1101/gr. 143000

[3] D. L. Cheo, S. A. Titus, D. N. R. Byrd, J. L. Hartley, G. F. Temple and M. A. Brasch, "Concerted Assembly and Cloning of Multiple DNA Segments Using in Vitro Site-Specific Recombination: Functional Analysis of Multi-Segment Expression Clones," Genome Research, Vol. 14, 2004, pp. 2111-2120. doi:10.1101/gr.2512204

[4] M. Karimi, B. De Meyer and P. Hilson, "Modular Cloning in Plant Cells," Trends in Plant Science, Vol. 10, 2005, pp. 103-105. doi:10.1016/j.tplants.2005.01.008

[5] M. Karimi, A. Depicke and P. Hilson, "Recombinational Cloning with Plant Gateway Vectors," Plant Physiology, Vol. 145, No. 4, 2007, pp. 1144-1154. doi:10.1104/pp.107.106989

[6] M. Karimi, D. Inze and A. Depicker, "Gateway Vectors for Agrobacterium-Mediated Plant Transformation," Trends in Plant Science, Vol. 7, 2002, pp. 193-195. doi:10.1016/S1360-1385(02)02251-3

[7] M. Karimi, A. Bleys, R. Vanderheghen and P. Hilson, "Building Blocks for Plant Gene Assembly," Plant Physiology, Vol. 145, No. 4, 2007, pp. 1183-1191. doi:10.1104/pp.107.110411

[8] M. D. Curtis andU. Grossniklaus, "A Gateway Cloning Vector Set for High-Throughput Functional Analysis of Genes in Planta," Plant Physiology, Vol. 133, No. 2, 2003, pp. 462-469. doi:10.1104/pp.103.027979

[9] K. W. Earley, J. R. Haag, O. Pontes, K. Opper, T. Juehne, K. Song and C. S. Pikkard, "Gateway-Compatible Vectors for Plant Functional Genomics and Proteomics," Plant Journal, Vol. 45, No. 4, 2006, pp. 616-629. doi:10.1111/j.1365-313X.2005.02617.X

[10] L.-Y. Lee and S. B. Gelvin, "T-DNA Binary Vectors and Systems," Plant Physiology, Vol. 146, No. 2, 2008, pp. 325-332. doi:10.1104/pp.107.113001

[11] A. Hoekema, P. R. Hirsch, P. J. J. Hooykaas and R. A. Schilperoort, "A Binary Plant Vector Strategy Based on Separation of vir- and T-Region of the Agrobacterium tumefaciens Ti-Plasmid," Nature, Vol. 303, No. 5913, 
1983, pp. 179-180. doi:10.1038/303179a0

[12] F. Bolivar, "Construction and Characterization of New Cloning Vehicles III: Derivatives of Plasmid pBR322 Carrying Unique EcoRI Sites for Selection of EcoRI Generated Recombinant DNA Molecules," Gene, Vol. 4, No. 2, 1979, pp. 121-136. doi:10.1016/0378-1119(78)90025-2

[13] J. G. Sutcliffe, "Complete Nucleotide Sequence of the Escherichia coli Plasmid pBR322," Cold Spring Harbor Symposium of Quantitative Biology, Vol. 43, 1978, pp. 77-90.

[14] J. G. Sutcliffe, "Nucleotide Sequence of the Ampicillin Resistance Gene of Escherichia coli Plasmid pBR322," Proceedings of the National Academy of Science of the United States of America, Vol. 75, 1978, pp. 3737-3741.

[15] S. P. Chambers, S. E. Prior, D. A. Barstow and N. P. Minton, "The pMTLnic Cloning Vectors I. Improved pUC Polylinker Regions to Facilitate the Use of Sonicated DNA for Nucleotide Sequencing," Gene, Vol. 68, No. 1, 1998, pp. 139-149. doi:10.1016/0378-1119(88)90606-3

[16] C. Yanisch-Perron, J. Vieira and J. Messing, "Improved M13 Phage Cloning Vectors and Host Strains: Nucleotide Sequences of the M13mpl8 and pUC19 Vectors," Gene, Vol. 33, No. 1, 1985, pp. 103-119. doi:10.1016/0378-1119(85)90120-9

[17] F. Barany, "Single Stranded Hexameric Linkers: A System for In-Phase Insertion Mutagenesis and Protein Engineering," Gene, Vol. 37, No. 1-3, 1985, pp. 111-123. doi:10.1016/0378-1119(85)90263-X

[18] H. C. Birnboim and J. Doly, "A Rapid Alkaline Extraction Procedure for Screening Recombinant Plasmid DNA," Nucleic Acids Research, Vol. 7, No. 6, 1979, pp. 1513-1523. doi:10.1093/nar/7.6.1513

[19] J. B. Hansen and R. H. Olsen, "Isolation of Large Bacterial Plasmids and Characterization of the P2 Incompatibility Group Plasmids pMG1 and pMG5," Journal of Bacteriology, Vol. 135, No. 1, 1978, pp. 227-238.

[20] B. Vogelstein and D. Gillespie, "Preparative and Analytical Purification of DNA from Agarose," Proceedings of the National Academy of Science of the United States of America, Vol. 76, 1979, pp. 615-619.
[21] J. Sambrook and D. W. Russell, "Molecular Cloning," Cold Spring Harbor Laboratory Press, Cold Spring Harbor, 2001.

[22] S. Lee, "New Binary Ti Vectors with the Co-Directional Replicons for Agrobacterium tumefaciens-Mediated Transformation of Higher Plants," $\mathrm{PhD}$ thesis, Louisiana State University, Baton Rouge, 2010.

[23] R. Hofgen R and L. Willmitzer, "Storage of Competent Cells for Agrobacterium tumefaciens," Nucleic Acids Research, Vol. 16, No. 20, 1988, p. 9822.

[24] G. Su, S. Park, S. Lee and N. Murai, "Low Co-Cultivation Temperature at $20^{\circ} \mathrm{C}$ Resulted in the Reproducible Maximum Increase in Both the Fresh Weight Yield and Stable Expression of GUS Activity after Agrobacterium tumefaciens-Mediated Transformation of Tobacco Leaf Disks," American Journal of Plant Sciences, Vol. 3, 2012, pp. 537-545. doi:10.4236/ajps.2012.34064

[25] S. Lee, G. Su, E. Lasserre, M. A. Aghazadeh and N. Murai, "Smaller High-Yielding Binary Ti Vectors pLSU with Co-Directional Replicons for Agrobacterum tumefaciens-Mediated Transformation of Higher Plants," Plant Science, Vol. 187,2012, pp. 49-58.

[26] S. B. Levy, L. M. MCMurry, T. M. Barbosa, V. Burdett, P. Courvalin, W. Hillen, M. C. Roberts, J. I. Rood and D. E. Taylor, "Nomenclature for New Tetracycline Resistance Determinants," Antimicrobial Agents and Chemotherapy, Vol. 43, No. 6, 1999, pp. 1523-1524.

[27] L. Brand, M. Horler, E. Nuesch, S. Vassalli, P. Barrell, W. Yang, R. A. Jefferson, U. Grossniklaus and M. D. Curtis, "A Versatile and Reliable Two-Component System for Tissue-Specific Gene Induction in Arabidopsis," Plant Physiology, Vol. 14, No. 4, 2006, pp. 1194-1204. doi:10.1104/pp.106.081299

[28] R. Xu and Q. Q. Li, "Protocol: Streamline Cloning of Genes into Binary Vectors in Agrobacterium via the Gateway TOPO Vector System," Plant Methods, Vol. 4, 2008, p. 4. doi:10.1186/1746-4811-4-4

[29] A. Himmelbach, U. Zierold, G. Hensel, J. Riechen, D. Douchkov, P. Schweizer and J. Kumiehn, "A Set of Modular Binary Vectors for Transformation of Cereals," Plant Physiology, Vol. 145, No. , 2007, pp. 1192-1200.

\section{Abbreviations}

A. media, Agrobacterium media;

FW: fresh weight;

GUS: $\beta$-glucuronidase;

REP and STA: the replication and stability region of VS1 replicon, respectively. 